\title{
QUASICOMPLETE FACTORIZATIONS OF RATIONAL MATRIX FUNCTIONS
}

\author{
R.A. Zuidwijk
}

It is shown that within the class of $n \times n$ rational matrix functions which are analytic at infinity with value $W(\infty)=I_{n}$, any rational matrix function $W$ is the product $W=W_{1} \cdots W_{\rho}$ of rational matrix functions $W_{1}, \ldots, W_{\rho}$ of McMillan degree one. Furthermore, such a factorization can be established with a number of factors not exceeding $2 \delta(W)-1$, where $\delta(W)$ denotes the McMillan degree of $W$.

\section{Introduction}

We study factorizations of complex rational $n \times n$ matrix functions $W$ which are analytic at infinity with value $W(\infty)=I_{n}$. Let us first consider the case when $n=1$; the scalar case. The rational scalar functions $w$ under consideration are the quotient of two monic polynomials $a^{\times}$and $a$ of the same degree: $w(\lambda)=a^{\times}(\lambda) / a(\lambda)$. We may assume that $a^{\times}$and $a$ are relative prime. The McMillan degree $\delta(w)$ of $w$ is then defined equal to the degree, say $m$, of the polynomials $a^{\times}$and $a$. The main theorem of algebra gives that

$$
w(\lambda)=\left(\frac{\lambda-\alpha_{1}^{\times}}{\lambda-\alpha_{1}}\right) \cdots\left(\frac{\lambda-\alpha_{m}^{\times}}{\lambda-\alpha_{m}}\right) .
$$

In other terms, $w$ is the product of $\delta(w)$ factors of McMillan degree one.

Also in the matrix case, i.e., in the case when $n>1$, the McMillan degree $\delta(W)$ of a rational $n \times n$ matrix function $W$ can be defined, and factorizations within the class of rational matrix functions can be studied. In fact, such factorizations arise in mathematical systems theory and have received considerable attention; see [1] and references given there. In Section 2 of this paper, a short discussion on the subject is given.

In contrast to the scalar case, not all rational matrix functions $W$ are the product of $\delta(W)$ factors of McMillan degree one. For example, the rational $2 \times 2$ matrix function

$$
W(\lambda)=\left(\begin{array}{cc}
1 & -\frac{1}{\lambda^{2}} \\
0 & 1
\end{array}\right)
$$


has McMillan degree $\delta(W)=2$, but requires at least three factors of McMillan degree one (see Example 4.3). Note that we require the factors to be rational matrix functions with an invertible value at infinity. If one gives up this restriction and allows for factorizations into possibly non-square factors, minimal factorizations into factors of McMillan degree one always can be achieved; see [16].

For two $n \times n$ rational matrix functions $W_{1}$ and $W_{2}$, the McMillan degree satisfies the sublogarithmic property $\delta\left(W_{1} W_{2}\right) \leq \delta\left(W_{1}\right)+\delta\left(W_{2}\right)$. Therefore, if $W=W_{1} \cdots W_{N}$ is a factorization into factors of McMillan degree one, we get $\delta(W) \leq \sum_{j=1}^{N} \delta\left(W_{j}\right)=N$. In other words, the number of elementary factors, i.e., factors of McMillan degree one, for a given rational matrix function $W$ is bounded from below by its McMillan degree $\delta(W)$. The main result of this paper, Theorem 4.2, gives an upper bound. It is shown that each rational matrix function $W$ can be factorized into a number of elementary factors not exceeding $2 \delta(W)-1$. In the forthcoming paper [6] this upper bound is proved to be sharp in a certain sense (see Remark 3 in the last section of this paper). The theorem is constructive; elements of pole assignment (see [12]) and of simultaneous reduction to complementary triangular forms (see Section 3) provide a method to obtain a factorization into elementary factors for a given rational matrix function.

So each rational matrix function is the product of a finite number of elementary rational matrix functions. For a given rational matrix function $W$, the minimal number of elementary factors is denoted by $\rho(W)$. A factorization of $W$ into $\rho(W)$ elementary factors is called a quasicomplete factorization. The notion complete factorization is reserved for factorizations of $W$ into $\delta(W)$ elementary factors (see [1]). Observe that a complete factorization is quasicomplete, that all rational matrix functions admit a quasicomplete factorization by Theorem 4.2, and that not all rational matrix functions admit a complete factorization by Example 4.3.

We conclude the introduction by some remarks on notation used in this paper. Throughout this paper, we shall identify a matrix with its action as a linear mapping relative to the standard bases. For an $n \times m$ matrix $B$, we denote its nullspace by Ker $B$ and its range by $\operatorname{Ran} B$. The linear span (in a given linear space) of a set $\mathcal{S}$ is denoted by span $\mathcal{S}$. Let $T=\left(T_{i j}\right)_{i, j=1}^{m}$ be an $m \times m$ matrix, then $\sigma(T)$ denotes the spectrum of $T$, i.e., the set of eigenvalues of $T$ in the complex plane $\mathbb{C}$. The complement $\rho(T)=\mathbb{C} \backslash \sigma(T)$ of the spectrum is called the resolvent set. Further, we define $\operatorname{diag}(T)=\left(T_{11}, \ldots, T_{m m}\right)^{T}$ to be the diagonal vector of $T$. A vector $\left(\tau_{1}, \ldots, \tau_{m}\right)^{T}$ in $\mathbb{C}^{m}$ is called a spectral vector of $T$ if $\operatorname{det}\left(\lambda I_{m}-T\right)=\prod_{j=1}^{m}\left(\lambda-\tau_{j}\right)$. The $n \times n$ identity matrix is denoted by $I_{n}$, while the symbol $O$ stands for a rectangular matrix with zero entries, the size of which is determined by the context.

\section{Rational Matrix Functions}

In this section, known material from systems theory pertinent to the main result of this paper (Theorem 4.2) is reviewed. For more background information, the reader is referred to [1] and [2] and the references given there.

An $n \times n$ rational matrix function $W: \lambda \mapsto\left(w_{i j}(\lambda)\right)_{i, j=1}^{n}$ is an $n \times n$ matrix with rational functions $w_{i j}$ as its entries. In this paper, all rational $n \times n$ matrix functions $W$ are 
assumed analytic at infinity with value $W(\infty)=I_{n}$. From systems theory it is known (see for example Theorem 2.2 in [1]) that such a matrix function $W$ can be written in the form

$$
W(\lambda)=I_{n}+C\left(\lambda I_{m}-A\right)^{-1} B, \quad \lambda \in \rho(A),
$$

where the matrices $A, B$ and $C$ are of the appropriate sizes. An expression of the form (1) is called a realization of $W$. With the realization (1), we associate a node $(A, B, C ; m, n)$. The first three entries in this tuple provide the three realization matrices, while the last two entries are integers denoting the dimensions of the spaces on which these matrices act.

The smallest possible integer $m$ for which $W$ admits a realization (1) is called the McMillan degree of $W$ and is denoted by $\delta(W)$. A realization (1) for which $m=\delta(W)$ is called a minimal realization. The node associated with a minimal realization is called a minimal node. An equivalent requirement for (1) to be a minimal realization (or its associated node to be minimal) is that the pair of matrices $A, B$ satisfies the controllability condition

$$
\operatorname{Ran} B+\operatorname{Ran}(A B)+\cdots+\operatorname{Ran}\left(A^{m-1} B\right)=\mathbb{C}^{m},
$$

and that the pair of matrices $C, A$ satisfies the observability condition

$$
\operatorname{Ker} C \cap \operatorname{Ker}(C A) \cap \cdots \cap \operatorname{Ker}\left(C A^{m-1}\right)=(0) \text {. }
$$

Note that a rational matrix function $W \equiv I_{n}$ if and oniy if $\delta(W)=0$. Recall that by the state space isomorphism theorem ([1], Theorem 3.1), all minimal nodes for a given rational matrix function are mutually similar: If $(A, B, C ; m, n)$ and $(F, G, H ; m, n)$ are two minimal nodes for the rational matrix function $W$, then there exists an invertible $m \times m$ matrix $S$, such that $S^{-1} A S=F, S^{-1} B=G$, and $C S=H$. If (1) is a (not necessarily minimal) realization for $W$, then the inverse $W^{-1}$ is given by the realization

$$
W(\lambda)^{-1}=W^{-1}(\lambda)=I_{n}-C\left(\lambda I_{m}-A^{\times}\right)^{-1} B, \quad \lambda \in \rho(A) \cap \rho\left(A^{\times}\right),
$$

where $A^{\times}=A-B C$. As a consequence, we get $\delta\left(W^{-1}\right)=\delta(W)$.

We now turn to factorizations of rational matrix functions. If $W_{1}, W_{2}$ and $W$ are rational matrix functions, then $W=W_{1} W_{2}$ denotes a factorization of $W$. If the minimal realizations of the factors are given by $W_{j}(\lambda)=I_{n}+C_{j}\left(\lambda I_{m_{j}}-A_{j}\right)^{-1} B_{j}$ for $j=1,2$, then the realization

$$
\begin{gathered}
W(\lambda)=W_{1}(\lambda) W_{2}(\lambda)= \\
I_{n}+\left(\begin{array}{ll}
C_{1} & C_{2}
\end{array}\right)\left[\lambda I_{m_{1}+m_{2}}-\left(\begin{array}{cc}
A_{1} & B_{1} C_{2} \\
O & A_{2}
\end{array}\right)\right]^{-1}\left(\begin{array}{c}
B_{1} \\
B_{2}
\end{array}\right)
\end{gathered}
$$

is called a product realization of $W$. Observe that the McMillan degree satisfies the sublogarithmic property

$$
\delta(W) \leq m_{1}+m_{2}=\delta\left(W_{1}\right)+\delta\left(W_{2}\right)
$$

In the case when $\delta(W)=\delta\left(W_{1}\right)+\delta\left(W_{2}\right)$, i.e., when the product realization (2) is a minimal realization of $W$, the factorization $W=W_{1} W_{2}$ is called a minimal factorization. 
A rational matrix function of McMillan degree one is called an elementary rational matrix function. A minimal realization of an elementary rational matrix function $W$ is of the form $W(\lambda)=I_{n}+(\lambda-\alpha)^{-1} c b^{T}$, where $\alpha$ is a complex number, and $c b^{T}$ is an $n \times n$ matrix of rank one ( $b$ and $c$ are column vectors here). The inverse of $W$ can be written as $W(\lambda)^{-1}=I_{n}-\left(\lambda-\alpha^{\times}\right)^{-1} c b^{T}$, where $\alpha^{\times}=\alpha-b^{T} c$.

Let $W$ be an $n \times n$ rational matrix function with minimal realization as in (1), so $\delta(W)=m$. If $W$ admits a factorization $W=W_{1} \cdots W_{m}$ where $W_{1}, \ldots, W_{m}$ are elementary rational matrix functions, we say that $W$ admits a complete factorization. Note that a complete factorization is minimal. A necessary and sufficient condition for complete factorization of a rational matrix function in terms of its realization matrices is given by the following theorem (Theorem 1.6 in [1] or Theorem 6.1 in [2]).

Theorem 2.1 Let $W$ be a rational $n \times n$ matrix function with minimal realization (1). Then $W$ admits a complete factorization if and only if there exists an invertible $m \times m$ matrix $S$ such that $S^{-1} A S$ is an upper triangular matrix and $S^{-1} A^{\times} S$ is a lower triangular matrix.

\section{Complementary Triangular Forms}

Theorem 2.1 in the previous section motivates the study of the following property:

A pair of $m \times m$ matrices $A, Z$ admits simultaneous reduction to complementary triangular forms if there exists an invertible $m \times m$ matrix $S$ such that $S^{-1} A S$ is an upper triangular matrix and $S^{-1} Z S$ is a lower triangular matrix.

Clearly, not all pairs of $m \times m$ matrices $A, Z$ have this property; for example, consider the case when $A=Z$ is nondiagonable. The collection of all pairs of $m \times m$ matrices that admit simultaneous reduction to complementary forms has been studied by various authors (cf. [2], [7], [8], [9], [11] and [17]).

In this section, we discuss briefly two known results on simultaneous reduction to complementary triangular forms. Then, a new result on complementary triangular forms is presented, which involves the notion of a spectral polynomial. We mention the following theorem which was first stated in terms of complete factorization of rational matrix functions [1]. We state the result as it appeared in [2].

Theorem 3.1 Let $A$ and $Z$ be $m \times m$ matrices. If either $A$ or $Z$ is diagonable, then the pair $A, Z$ admits simultaneous reduction to complementary triangular forms.

Most results on simultaneous reduction to complementary triangular forms are concerned with pairs of matrices which belong to certain classes of matrices. As an example, we will give a result for pairs of first companion matrices. Recall that a first companion matrix is of the form (see also [13]) 


$$
C_{a}=\left(\begin{array}{ccccc}
0 & 1 & 0 & \cdots & 0 \\
\vdots & 0 & \ddots & \ddots & \vdots \\
\vdots & & \ddots & 1 & 0 \\
0 & \cdots & \cdots & 0 & 1 \\
-a_{0} & -a_{1} & \cdots & -a_{m-2} & -a_{m-1}
\end{array}\right)
$$

where $a_{0}, \ldots, a_{m-1}$ are complex numbers. Note that there is a one-to-one correspondence between monic polynomials of degree $m$ and first companion matrices, given by the equation

$$
\operatorname{det}\left(\lambda-C_{a}\right)=a_{0}+a_{1} \lambda+\cdots+a_{m-1} \lambda^{m-1}+\lambda^{m} .
$$

The following theorem, which describes simultaneous reduction to complementary triangular forms for pairs of first companion matrices, is taken from [2], Theorem 3.2.

Theorem 3.2 Let $A$ and $Z$ be first companion $m \times m$ matrices. The pair $A, Z$ admits simultaneous reduction to complementary triangular forms if and only if there exist spectral vectors $\left(\alpha_{1}, \ldots, \alpha_{m}\right)^{T}$ and $\left(\zeta_{1}, \ldots, \zeta_{m}\right)^{T}$ of $A$ and $Z$, respectivily, such that

$$
\alpha_{k} \neq \zeta_{l}, \quad k+l \leq m
$$

In the remaining part of this section, we state and prove Theorem 3.3, which makes use of and is an extension of Theorem 3.1. Moreover, the theorem provides an important step in the proof of the main result of this paper. The theorem requires the notion of a spectral polynomial explained now: Let $B$ be an $m \times m$ matrix and let the mutually distinct eigenvalues of $B$ be denoted by $\beta_{1}, \ldots, \beta_{s}$. Define the spectral polynomial of $B$ by

$$
p_{B}(\lambda)=\left(\lambda-\beta_{1}\right) \cdots\left(\lambda-\beta_{s}\right) .
$$

This polynomial is the monic polynomial of minimal degree vanishing on the spectrum of $B$. Note that the matrix $p_{B}(B)$ is always nilpotent and that $p_{B}(B)=O_{m}$ if and only if $B$ is diagonable. In fact, the subspace $\operatorname{Ker} p_{B}(B)$ is the linear span of all eigenvectors of $B$.

Theorem 3.3 Let $A$ and $Z$ be $m \times m$ matrices. If either

$$
\operatorname{Ker} p_{A}(A)+\operatorname{Ker} p_{Z}(Z)=\mathbb{C}^{m}
$$

or

$$
\operatorname{Ran} p_{A}(A) \cap \operatorname{Ran} p_{Z}(Z)=(0),
$$

then the pair $A, Z$ admits simultaneous reduction to complementary triangular forms.

The subspace $\operatorname{Ker} p_{A}(A)+\operatorname{Ker} p_{Z}(Z)$ in (5) is the linear span of the eigenvectors of $A$ and the eigenvectors of $Z$. Since the eigenvectors of a diagonable matrix span the whole space, it follows that Theorem 3.1 is a special case of Theorem 3.3. Before proving the theorem, we first give a lemma. 
Lemma 3.4 Let $A_{1}$ and $Z_{1}$ be $m_{1} \times m_{1}$ matrices, $A_{2}$ and $Z_{2}$ be $m_{2} \times m_{2}$ matrices, $A_{12}$ an $m_{1} \times m_{2}$ matrix, $Z_{21}$ an $m_{2} \times m_{1}$ matrix. Define $m=m_{1}+m_{2}$ and consider the $m \times m$ matrices

$$
A=\left(\begin{array}{cc}
A_{1} & A_{12} \\
O & A_{2}
\end{array}\right), \quad Z=\left(\begin{array}{cc}
Z_{1} & 0 \\
Z_{21} & Z_{2}
\end{array}\right) .
$$

If both pairs $A_{1}, Z_{1}$ and $A_{2}, Z_{2}$ admit simultaneous reduction to complementary forms, then the pair $A, Z$ has the same property.

Proof By assumption, there exist invertible matrices $S_{1}$ and $S_{2}$, such that $S_{1}^{-1} A_{1} S_{1}$ and $S_{2}^{-1} A_{2} S_{2}$ are upper triangular and $S_{1}^{-1} Z_{1} S_{1}$ and $S_{2}^{-1} Z_{2} S_{2}$ are lower triangular. Put $S=S_{1} \oplus S_{2}$ and verify that $S^{-1} A S$ is upper triangular and $S^{-1} Z S$ is lower triangular.

Proof of Theorem 3.3 We first prove that (5) implies that the pair $A, Z$ admits simultaneous reduction to complementary triangular forms. Write $\operatorname{dim} \operatorname{Ker} p_{A}(A)=k$ and $\operatorname{dim} \operatorname{Ker} p_{Z}(Z)=l$. There exist vectors $\phi_{1}, \ldots, \phi_{k}$ and complex numbers $\alpha_{1}, \ldots, \alpha_{k}$ such that

$$
\operatorname{Ker} p_{A}(A)=\operatorname{span}\left\{\phi_{1}, \ldots, \phi_{k}\right\}, \quad A \phi_{i}=\alpha_{i} \phi_{i}, \quad i=1, \ldots, k,
$$

and there exist vectors $\psi_{1}, \ldots, \psi_{l}$ and complex numbers $\zeta_{1}, \ldots, \zeta_{l}$ such that

$$
\operatorname{Ker} p_{Z}(Z)=\operatorname{span}\left\{\psi_{1}, \ldots, \psi_{l}\right\}, \quad Z \psi_{j}=\zeta_{j} \dot{\psi}_{j}, \quad j=1, \ldots, l
$$

Then (5) implies that $k+l \geq m$ and that the vectors $\dot{\phi}_{1}, \ldots, \phi_{k}, \psi_{1}, \ldots, \psi_{l}$ span the whole space $\mathbb{C}^{m}$. From this collection of vectors, a basis for $\mathbb{C}^{m}$ can be selected: There exist an integer $0 \leq s \leq m$, a strictly increasing mapping $\pi:\{1, \ldots, s\} \longrightarrow\{1, \ldots, k\}$ and a strictly increasing mapping $\rho:\{1, \ldots, m-s\} \longrightarrow\{1, \ldots, l\}$ such that $\phi_{\pi(1)}, \ldots, \phi_{\pi(s)}, \psi_{\rho(1)}, \ldots, \psi_{\rho(m-s)}$ is a basis for $\mathbb{C}^{m}$. With respect to this basis, $A$ and $Z$ assume the forms

$$
A=\left(\begin{array}{cc}
D_{1} & A_{12} \\
O & A_{22}
\end{array}\right), \quad Z=\left(\begin{array}{cc}
Z_{11} & 0 \\
Z_{21} & D_{2}
\end{array}\right),
$$

where $D_{1}$ is an $s \times s$ diagonal matrix with diagonal vector $\left(\alpha_{\pi(1)}, \ldots, \alpha_{\pi(s)}\right)^{T}$ and $D_{2}$ is an $(m-s) \times(m-s)$ diagonal matrix with diagonal vector $\left(\zeta_{\rho(1)}, \ldots, \zeta_{\rho(m-s)}\right)^{T}$. By Theorem 3.1 and Lemma 3.4 it now follows that the pair $A, Z$ admits simultaneous reduction to complementary triangular forms.

Next, we prove that (6) implies that the pair $A, Z$ admits simultaneous reduction to complementary triangular forms. First note that $(6)$ is equivalent to

$$
\operatorname{Ker} p_{A^{*}}\left(A^{*}\right)+\operatorname{Ker} p_{Z^{*}}\left(Z^{*}\right)=\mathbb{C}^{m}
$$

By the argument given in the first part of the proof, it follows that the pair $A^{*}$ and $Z^{*}$ admits simultaneous reduction to complementary triangular forms. It easily follows that the pair $A, Z$ has the same property. The theorem is proved. 
The sufficient conditions (5) and (6) in Theorem 3.3 are easily seen not to be necessary: Consider the pair of $3 \times 3$ matrices

$$
A=\left(\begin{array}{lll}
0 & 1 & 0 \\
0 & 0 & 1 \\
0 & 0 & 0
\end{array}\right), \quad Z=\left(\begin{array}{lll}
0 & 0 & 0 \\
1 & 0 & 0 \\
0 & 1 & 0
\end{array}\right)
$$

It follows that $p_{A}(\lambda)=p_{Z}(\lambda)=\lambda$, and that the conditions (5) and (6) are not fulfilled. On the other band, the pair of matrices $A, Z$ are in complementary triangular forms.

\section{Factorizations into Elementary Factors}

As demonstrated in the introduction, rational scalar functions always admit a complete factorization. In general, however, rational matrix functions need not admit a complete factorization. Indeed, for each pair of $m \times m$ matrices $A, Z$, one can construct a minimal realization $W(\lambda)=I_{n}+C\left(\lambda I_{m}-A\right)^{-1} B$ such that $Z=A^{\times}=A-B C$ (for details, see Theorem 5.1 in [2]). Hence if the pair $A, Z$ does not admit simultaneous reduction to complementary triangular forms, then the minimal realization $W(\lambda)=I_{n}+C\left(\lambda I_{m}-A\right)^{-1} B$ does not admit a complete factorization. For this reason, more relaxed notions of factorization into elementary factors have been considered. In [16], it is shown that each rational matrix function admits a minimal factorization into possibly non-square elementary rational matrix functions. In Theorem 4.2, the main result of this paper, it will be proved that each rational matrix function (1) is the product of elementary rational matrix functions, i.e., is of the form

$$
W=W_{1} \cdots W_{N}
$$

where $W_{1}, \ldots, W_{N}$ again are elementary rational matrix functions, but where the number of factors $N$ may exceed the McMillan degree of $W$.

Let $W$ be a rational matrix function, and let $\rho(W)$ denote the infimum of all integers $N$, such that $W$ admits a factorization into $N$ elementary rational matrix functions. In Theorem 4.2 below, it is shown that $\rho(W)<\infty$, i.e., that all rational matrix function admit a factorization into a finite number of elementary rational matrix functions. A factorization involving the minimal number $\rho=\rho(W)$ of elementary factors

$$
W=W_{1} \cdots W_{\rho}
$$

is called a quasicomplete factorization. As explained in the introduction, $\rho(W) \geq \delta(W)$.

Let $W$ be a rational matrix function with minimal node $(A, B, C ; m, n)$ and let $N \geq m$ be an integer. The tuple $(\hat{A}, \hat{B}, \hat{C} ; N, n)$ is called a dilated node of the node $(A, B, C ; m, n)$, if it is of the form

$$
\hat{A}=\left(\begin{array}{ccc}
A_{1} & A_{12} & A_{13} \\
O & A & A_{23} \\
O & O & A_{33}
\end{array}\right), \quad \hat{B}=\left(\begin{array}{c}
B_{1} \\
B \\
O
\end{array}\right), \quad \hat{C}=\left(\begin{array}{lll}
O & C & C_{3}
\end{array}\right),
$$


where $A_{1}$ is a $\kappa \times \kappa$ matrix for some integer $0 \leq \kappa \leq N-m, A_{33}$ is an $(N-m-\kappa) \times(N-m-\kappa)$ matrix, and $A_{12}, A_{13}$ and $A_{23}$ are of corresponding sizes. Further, $B_{1}$ is a $\kappa \times n$ matrix, and $C_{3}$ is an $n \times(N-m-\kappa)$ matrix. By computation, one can verify that

$$
W(\lambda)=I_{n}+\hat{C}\left(\lambda I_{N}-\hat{A}\right)^{-1} \hat{B},
$$

so that the tuple $(\hat{A}, \hat{B}, \hat{C} ; N, n)$ indeed is a node for $W$. Observe that in the case when $N>m$, the realization in (8) is not minimal. The following lemma shows how the notion of a dilated node is used to study factorizations into elementary factors.

Lemma 4.1 Let $W$ be a rational $n \times n$ matrix function with minimal node $(A, B, C ; m, n)$, and let $N \geq m$ be an integer. Then $W$ admits a factorization $W=W_{1} \cdots W_{N}$ into $N$ elementary factors if and only if there exists a dilated node $(\hat{A}, \hat{B}, \hat{C} ; N, n)$ of $(A, B, C ; m, n)$ such that the pair of $N \times N$ matrices $\hat{A}, \hat{A}^{\times}=\hat{A}-\hat{B} \hat{C}$ admits simultaneous reduction to complementary triangular forms.

Proof To prove the only if part, assume that $W$ admits a factorization as in (7). Denote the product realization by $W(\lambda)=I_{n}+\tilde{C}\left(\lambda I_{N}-\tilde{A}\right)^{-1} \tilde{B}$. Then $\tilde{A}$ is upper triangular, and $\tilde{A}-\tilde{B} \tilde{C}$ is lower triangular: See the only if part of the proof of Theorem 6.1 in [1]. By Theorem 3.2 in [1], there exists an invertible $N \times N$ matrix $T$, such that $\left(T^{-1} \tilde{A} T, T^{-1} \tilde{B}, \tilde{C} T ; N, n\right)$ is a dilation of some minimal node $(F, G, H ; m, n)$ for $W$. The state space isomorphism theorem provides an invertible $m \times m$ matrix $V$ such that $V^{-1} F V=A$, $V^{-1} G=B$ and $H V=C$. Define the invertible $N \times N$ matrix (with the same block structure as $\hat{A})$

$$
Y=\left(\begin{array}{lll}
I & O & O \\
O & V & O \\
O & O & I
\end{array}\right),
$$

and write $S=T Y$. Then $\hat{A}=S^{-1} \tilde{A} S, \hat{B}=S^{-1} \tilde{B}$ and $\hat{C}=\tilde{C} S$ provide a dilated node as described in the lemma. The if part is proved in the same fashion as the if part of Theorem 6.1 in [2]).

We now come to the main result in this paper.

Theorem 4.2 Consider the minimal realization

$$
W(\lambda)=I_{n}+C\left(\lambda I_{m}-A\right)^{-1} B .
$$

Define the integer $k(W)=m-\operatorname{dim}\left(\operatorname{Ker} p_{A}(A)+\operatorname{Ker} p_{A^{\times}}\left(A^{\times}\right)\right)$, where $A^{\times}=A-B C$. Then the minimal number of elementary factors $\rho(W)$ for $W$ satisfies the estimate

$$
\rho(W) \leq \delta(W)+k(W) \leq 2 \delta(W)-1 .
$$


Proof The pair of matrices $A, B$ in the minimal realization (9) is controllable. Therefore, according to the spectral assignment theorem (Theorem 6.5.1 in [12]), there exists for each $m$-tuple of complex numbers $g_{1}, \ldots, g_{m}$ an $n \times m$ matrix $K$, such that $A+B K$ has eigenvalues $g_{1}, \ldots, g_{m}$. We will assume these eigenvalues to be distinct, so that $A+B K$ is diagonable. We will also assume that this set of complex numbers does not intersect $\sigma(A) \cup \sigma\left(A^{\times}\right)$.

Consider the subspace $M=\operatorname{Ker} p_{A}(A)+\operatorname{Ker} p_{A^{\times}}\left(A^{\times}\right)$. By definition, $\operatorname{codim} M=$ $k(W)$. Write $k=k(W)$, and note that $0 \leq k \leq m-1$. There exist $k$ eigenvectors $x_{1}, \ldots x_{k}$ for $A+B K$, such that $M \oplus \operatorname{span}\left\{x_{1}, \ldots, x_{k}\right\}=\mathbb{C}^{m}$. After renumbering the eigenvalues, we may write $(A+B K) x_{j}=g_{j} x_{j}$ for $j=1, \ldots, k$. Define the $m \times k$ matrix $X=\left(x_{1}, x_{2}, \ldots, x_{k}\right)$. Then $(A+B K) X=X G$, where $G$ is an $k \times k$ diagonal matrix with diagonal vector $\operatorname{diag}(G)=$ $\left(g_{1}, \ldots, g_{k}\right)^{T}$. Define the $n \times k$ matrix $F=-K X$ to obtain $A X-X G=B F$. Consider the matrices

$$
\hat{A}=\left(\begin{array}{cc}
A & B F \\
O & G
\end{array}\right), \quad \hat{B}=\left(\begin{array}{c}
B \\
O
\end{array}\right), \quad \hat{C}=\left(\begin{array}{cc}
C & F
\end{array}\right)
$$

Further, write

$$
\hat{A}^{\times}=\hat{A}-\hat{B} \hat{C}=\left(\begin{array}{cc}
A^{\times} & O \\
O & G
\end{array}\right)
$$

where, as usual, $A^{\times}=A-B C$. Note that

$$
\begin{aligned}
& \left(\begin{array}{cc}
I_{m} & -X \\
O & I_{k}
\end{array}\right)\left(\begin{array}{cc}
A & O \\
O & G
\end{array}\right)\left(\begin{array}{cc}
I_{m} & X \\
O & I_{k}
\end{array}\right)= \\
& \left(\begin{array}{cc}
A & A X-X G \\
O & G
\end{array}\right)=\left(\begin{array}{cc}
A & B F \\
O & G
\end{array}\right)=\hat{A} .
\end{aligned}
$$

Since $\sigma(A) \cap \sigma(G)=\emptyset$, we get $p_{\hat{A}}(\lambda)=p_{A}(\lambda) p_{G}(\lambda)$. Hence

$$
p_{\hat{A}}(\hat{A})=\left(\begin{array}{cc}
I_{m} & -X \\
O & I_{k}
\end{array}\right)\left(\begin{array}{cc}
p_{G}(A) & O \\
O & p_{A}(G)
\end{array}\right)\left(\begin{array}{cc}
p_{A}(A) & O \\
O & O_{k}
\end{array}\right)\left(\begin{array}{cc}
I_{m} & X \\
O & I_{k}
\end{array}\right),
$$

where $p_{G}(A)$ and $p_{A}(G)$ are invertible matrices. We have also used $p_{G}(G)=O_{k}$. Therefore,

$$
\operatorname{Ker} p_{\hat{A}}(\hat{A})=\left(\begin{array}{cc}
I_{m} & -X \\
O & I_{k}
\end{array}\right) \operatorname{Ker}\left(\begin{array}{cc}
p_{A}(A) & O \\
O & O_{k}
\end{array}\right) \text {. }
$$

Also,

$$
\operatorname{Ker} p_{\dot{A}^{\times}}\left(\hat{A}^{\times}\right)=\operatorname{Ker}\left(\begin{array}{cc}
p_{A^{\times}}\left(A^{\times}\right) & O \\
O & O_{k}
\end{array}\right)
$$

It is not difficult to verify, that

$$
\operatorname{Ker} p_{\hat{A}}(\hat{A})+\operatorname{Ker} p_{\hat{A}^{\times}}\left(\hat{A}^{\times}\right)=\mathbb{C}^{m+k}
$$


if and only if

$$
\text { Ker } p_{A}(A)+\operatorname{Ker} p_{A^{\times}}\left(A^{\times}\right)+\operatorname{Ran} X=M+\operatorname{Ran} X=\mathbb{C}^{m} .
$$

By construction, the latter is the case. Theorem 3.3 then yields that the pair $\hat{A}, \hat{A}^{\times}$admits simultaneous reduction to complementary triangular forms. Next, use Lemma 4.1 to obtain that $W$ admits a factorization as in (7), with $N=m+k=\delta(W)+k(W)$. The theorem is proved.

To illustrate Theorem 4.2 and its proof, we will factorize a given rational matrix function which does not admit a complete factorization into elementary factors. Such a factorization of this particular rational matrix function was already known to Thijsse [14].

Example 4.3 In this example, we will compute a quasicomplete factorization for the rational matrix function

$$
W(\lambda)=\left(\begin{array}{cc}
1 & \frac{-1}{\lambda^{2}} \\
0 & 1
\end{array}\right)
$$

with minimal realization

$$
W(\lambda)=I_{2}+C\left(\lambda I_{2}-A\right)^{-1} B
$$

where the realization matrices are given by

$$
A=\left(\begin{array}{ll}
0 & 1 \\
0 & 0
\end{array}\right), \quad B=\left(\begin{array}{ll}
0 & 0 \\
0 & 1
\end{array}\right), \quad C=\left(\begin{array}{cc}
-1 & 0 \\
0 & 0
\end{array}\right)
$$

Compute

$$
A^{\times}=A-B C=\left(\begin{array}{ll}
0 & 1 \\
0 & 0
\end{array}\right) .
$$

Since $A=A^{\times}$is nondiagonable, it follows that the pair $A, A^{\times}$does not admit simultaneous reduction to complementary triangular forms. By Theorem 2.1, $W$ does not admit a complete factorization, so $\rho(W)>2$. We will now follow the proof of Theorem 4.2. Note that with

$$
K=\left(\begin{array}{ll}
0 & 0 \\
1 & 0
\end{array}\right)
$$

we have

$$
A+B K=\left(\begin{array}{ll}
0 & 1 \\
1 & 0
\end{array}\right)
$$

so $A+B K$ is diagonable. Observe that $k(W)=1$, and take

$$
X=\left(\begin{array}{l}
1 \\
1
\end{array}\right), \quad G=1, \quad F=\left(\begin{array}{c}
0 \\
-1
\end{array}\right)
$$


so that $A X-X G=B F$. We now obtain the matrices

$$
\hat{A}=\left(\begin{array}{ccc}
0 & 1 & 0 \\
0 & 0 & -1 \\
0 & 0 & 1
\end{array}\right), \quad \hat{B}=\left(\begin{array}{ll}
0 & 0 \\
0 & 1 \\
0 & 0
\end{array}\right), \quad \hat{C}=\left(\begin{array}{ccc}
-1 & 0 & 0 \\
0 & 0 & -1
\end{array}\right)
$$

and

$$
\hat{A}^{\times}=\hat{A}-\hat{B} \hat{C}=\left(\begin{array}{lll}
0 & 1 & 0 \\
0 & 0 & 0 \\
0 & 0 & 1
\end{array}\right)
$$

By construction, the pair $\hat{A}, \hat{A}^{\times}$admits simultaneous reduction to complementary triangular forms. In fact, the similarity

$$
S=\left(\begin{array}{ccc}
1 & 1 & 0 \\
1 & 0 & 0 \\
-1 & 0 & 1
\end{array}\right)
$$

transforms $\hat{A}$ and $\hat{A}^{\times}$into complementary triangular forms as follows:

$$
S^{-1} \hat{A} S=\left(\begin{array}{ccc}
1 & 0 & -1 \\
0 & 0 & 1 \\
0 & 0 & 0
\end{array}\right), \quad S^{-1} \hat{A}^{\times} S=\left(\begin{array}{ccc}
0 & 0 & 0 \\
1 & 0 & 0 \\
-1 & 0 & 1
\end{array}\right) \text {. }
$$

In addition,

$$
S^{-1} \hat{B}=\left(\begin{array}{cc}
0 & 1 \\
0 & -1 \\
0 & 1
\end{array}\right), \quad \hat{C} S=\left(\begin{array}{ccc}
-1 & -1 & 0 \\
1 & 0 & -1
\end{array}\right)
$$

As explained in the proof of Theorem 6.1 in [2], we may now calculate the elementary factors explicitly and obtain $W(\lambda)=W_{1}(\lambda) W_{2}(\lambda) W_{3}$, with

$$
\begin{gathered}
W_{1}(\lambda)=\left(\begin{array}{ll}
1 & 0 \\
0 & 1
\end{array}\right)+\frac{1}{\lambda-1}\left(\begin{array}{c}
-1 \\
1
\end{array}\right)\left(\begin{array}{ll}
0 & 1
\end{array}\right)=\left(\begin{array}{cc}
1 & \frac{-1}{\lambda-1} \\
0 & \frac{\lambda}{\lambda-1}
\end{array}\right), \\
W_{2}(\lambda)=\left(\begin{array}{ll}
1 & 0 \\
0 & 1
\end{array}\right)+\frac{1}{\lambda}\left(\begin{array}{c}
-1 \\
0
\end{array}\right)\left(\begin{array}{cc}
0 & -1
\end{array}\right)=\left(\begin{array}{cc}
1 & \frac{1}{\lambda} \\
0 & 1
\end{array}\right), \\
W_{3}(\lambda)=\left(\begin{array}{ll}
1 & 0 \\
0 & 1
\end{array}\right)+\frac{1}{\lambda-1}\left(\begin{array}{c}
0 \\
-1
\end{array}\right)\left(\begin{array}{ll}
0 & 1
\end{array}\right)=\left(\begin{array}{cc}
1 & 0 \\
0 & \frac{\lambda-1}{\lambda}
\end{array}\right) .
\end{gathered}
$$

We may conclude that $\rho(W)=3$. 4.2 .

We finish this section with a number of remarks which are related to Theorem 
1. In the proof of Theorem 4.2, only the controllability of the pair $A, B$ is used explicitly. The observability of the pair $C, A$ enters in the proof through the minimality condition $m=\delta(W)$.

2. By taking adjoints and observing that $\delta(W)=\delta\left(W^{*}\right)$ and $\rho(W)=\rho\left(W^{*}\right)$, we can state a dual version of Theorem 4.2. Indeed,

$$
k^{*}(W)=\operatorname{codim}\left[\operatorname{Ker} p_{A^{*}}\left(A^{*}\right)+\operatorname{Ker} p_{\left(A^{*}\right)^{\times}}\left(\left(A^{*}\right)^{\times}\right)\right]=\operatorname{dim}\left[\operatorname{Ran} p_{A}(A) \cap \operatorname{Ran} p_{A^{\times}}\left(A^{\times}\right)\right]
$$

leads to $\rho(W) \leq \delta(W)+k^{*}(W) \leq 2 \delta(W)-1$. It follows that

$$
\rho(W) \leq \delta(W)+\min \left\{k(W), k^{*}(W)\right\}
$$

3. In the forthcoming paper [6], it is shown that the estimate $\rho(W) \leq 2 \delta(W)-1$ is sharp in the following sense: For each positive integer $m$, the rational matrix function

$$
W(\lambda)=\left(\begin{array}{cc}
1 & -\lambda^{-m} \\
0 & 1
\end{array}\right)
$$

satisfies $\delta(W)=m$ and $\rho(W)=2 m-1$.

4. The dilated node $(\hat{A}, \hat{B}, \hat{C} ; m+k, n)$ in the proof of Theorem 4.2 is of a special form. In fact, it is the product realization of the product $W I_{n}$ with realizations

$$
W(\lambda)=I_{n}+C\left(\lambda I_{m}-A\right)^{-1} B, \quad I_{n}=I_{n}+F\left(\lambda I_{k}-G\right)^{-1} O
$$

5. Let $W$ be an $n \times n$ rational matrix function, and let $\nu(W)$ denote the maximal number of non-trivial factors that can occur in a minimal factorization of $W$. Then $1 \leq \nu(W) \leq \delta(W)$. Write $\nu(W)=\nu$, and consider the minimal factorization $W=W_{1} \cdots W_{\nu}$. By Theorem 4.2, applied on all factors separately, we get

$$
\rho(W) \leq \sum_{j=1}^{\nu} \rho\left(W_{j}\right) \leq \sum_{j=1}^{\nu}\left[\delta\left(W_{j}\right)+k\left(W_{j}\right)\right] \leq \sum_{j=1}^{\nu}\left[2 \delta\left(W_{j}\right)-1\right]=2 \delta(W)-\nu(W),
$$

so $\rho(W)+\nu(W) \leq 2 \delta(W)$. This inequality is not sharp. Indeed, the rational matrix function $W(\lambda)=I_{3}+C\left(\lambda I_{3}-A\right)^{-1} B$, with

$$
A=\left(\begin{array}{lll}
0 & 1 & 0 \\
0 & 0 & 1 \\
0 & 0 & 0
\end{array}\right), \quad B=\left(\begin{array}{ccc}
0 & 1 & -1 \\
0 & 0 & 1 \\
1 & 0 & 0
\end{array}\right), \quad C=\left(\begin{array}{ccc}
0 & 0 & 0 \\
-1 & 1 & 0 \\
-1 & 0 & 1
\end{array}\right)
$$

satisfies $\delta(W)=3, \rho(W)=4$, and $\nu(W)=1$.

6. A companion based rational matrix function is a rational matrix function, which admits a minimal realization (1) with $A$ and $A^{\times}$first companion matrices. Complementary triangular forms for a pair of first companion matrices are now well understood; see Theorem 3.2. This 
result states that a pair of first companion matrices admits simultaneous reduction to complementary triangular forms if and only if a combinatorial condition is met. In this manner, complete factorization for companion based rational matrix functions is characterized. Surprisingly enough, an important job scheduling problem, known as the Two Machine Flow Shop Problem, can be rewritten in terms of this combinatorial condition. In fact, there exists a correspondence between instances of the Two Machine Flow shop Problem on the one hand and companion based rational matrix functions on the other hand. For more details, see [3], [4], [5] and [6]. In particular, we mention the fact that the minimum processing time performed by an optimale schedule for such a Two Machine Flow shop Problem is closely related to the positive integer $\rho(W)$ for the associated companion based rational matrix function $W$.

Addendum

After the completion of this paper, we learned about another method, which was proposed in [10], to factorize rational matrix functions into elementary factors. One of the results from [10] comes down to $\rho(W) \leq 2 \delta(W)-\max \left\{\operatorname{dim} \operatorname{Ker} p_{A}(A)\right.$, dim Ker $\left.p_{A^{\times}}\left(A^{\times}\right)\right\}$, while in this paper we arrive at the sharper estimate $\rho(W) \leq 2 \delta(W)-\operatorname{dim}\left(\operatorname{Ker} p_{A}(A)+\operatorname{Ker} p_{A^{\times}}\left(A^{\times}\right)\right)$. To illustrate that the latter inequality is sharper indeed, we give an example. Let

$$
W(\lambda)=\left(\begin{array}{cc}
\frac{\lambda^{2}-1}{\lambda^{2}} & \frac{1}{\lambda} \\
\frac{-1}{\lambda} & 1
\end{array}\right) .
$$

By the method in [10], the rational matrix function $W$ is multiplied to the left with an elementary rational matrix function $V$, such that $V W$ admits a complete factorization and $\delta(V W)=\delta(W)=2$. For example, take $V$ of the form $(\alpha \neq 0)$

$$
V(\lambda)=\left(\begin{array}{cc}
\frac{\lambda}{\lambda-\alpha} & 0 \\
0 & 1
\end{array}\right) .
$$

By construction, there exist elementary rational matrix functions $V_{1}$ and $V_{2}$, such that $V W=$ $V_{1} V_{2}$. One then gets $W=V^{-1} V_{1} V_{2}$. It turns out that $\delta\left(V^{-1} V_{1}\right)=2$, so that the factorization does not "collapse" into a complete one. By the methods presented in this paper, it follows immediately that $W$ does admit a complete factorization. Indeed, given a minimal realization $W(\lambda)=I_{2}+C\left(\lambda I_{2}-A\right)^{-1} B$, it is easy to verify that $\operatorname{Ker} A+\operatorname{Ker} A^{\times}=\mathbb{C}^{2}$.

Acknowledgement

It is a pleasure to thank $\mathrm{H}$. Bart for his stimulating remarks and comments.

\section{References}

[1] H. Bart, I. Gohberg, M.A. Kaashoek, Minimal Factorization of Matrix and Operator Functions, Operator Theory: Adv. Appl. 1, Birkhäuser Verlag, Basel (1979).

[2] H. Bart, H. Hoogland, Complementary Triangular Forms of Pairs of Matrices, Realizations with Prescribed Main Matrices, and Complete Factorization of Rational Matrix Functions, Lin. Alg. Appl. 103: 193-228 (1988).

[3] H. Bart, L.G. Kroon, Companion Based Matrix Functions: Description and Factorization, to appear in Lin. Alg. Appl. 
[4] H. Bart, L.G. Kroon, Factorization and Job Scheduling: a connection via Companion Based Rational Matrix Functions, to appear in Lin. Alg. Appl.

[5] H. Bart, L.G. Kroon, Variants of the Two Machine Flow Shop Problem connected with Factorization of Rational Matrix Functions, to appear in European Journal of Operational Research.

[6] H. Bart, L.G. Kroon, R.A. Zuidwijk, Quasicomplete Factorization and The Two Machine Flow Shop Problem, in preparation.

[7] H. Bart, G.Ph.A. Thijsse, Complementary Triangular Forms of Upper Triangular Toeplitz Matrices, Operator Theory: Adv. Appl. 40: 133-149 (1989).

[8] H. Bart, G.Ph.A. Thijsse, Complementary Triangular Forms of Nonderogatory, Jordan and Rank One Matrices, Report 9003/B, Econometric Institute, Erasmus University Rotterdam (1990).

[9] H. Bart, H.K: Wimmer, Simultaneous Reduction to Triangular and Companion Forms of Pairs of Matrices: The Case $\operatorname{rank}(I-A Z)=1$, Lin. Alg. Appl. 150: 443-461 (1991).

[10] P. DeWilde, J.P. VandeWalle, On the Factorization of a Nonsingular Rational Matrix, IEEE Tr. Circuits and Systems, vol. 22, no. 8: 637-645 (1975).

[11] S. Friedland, Pairs of Matrices Which Do Not Admit a Complementary Triangular Form, Lin. Alg. Appl. 150: 119-123 (1990).

[12] I. Gohberg, P. Lancaster, L. Rodman, Invariant Subspaces of Matrices with Applications, J. Wiley and Sons, New York (1986).

[13] P. Lancaster, M. Tismenetsky, The Theory of Matrices, Second Edition with Applications, Academic Press, Orlando, Fl. (1985).

[14] G.Ph.A. Thijsse, personal communication.

[15] G.Ph.A. Thijsse, Eigenspace and Jordan-Chain Techniques for the Description of Complementary Triangular Forms, submitted.

[16] S.H. Tan, J. Vandewalle, On Factorizations of Rational Matrices, IEEE Transactions on Circuits and Systems 35: 1179-1181 (1988).

[17] R.A. Zuidwijk, Complementary Triangular Forms for Pairs of Matrices and Operators, doctoral thesis (1994).

Center for Mathematics and Information Science

P.O. Box 94079 , 1090 GB Amsterdam

The Netherlands

E-mail: zuidwijk@cwi.nl

AMS 1991 Subject Classification 15A21, 15A23, 47A68, 93B15, 93B25.

Submitted: May 17, 1995

Revised: April 15, 1996 